\title{
Functional Respiratory Imaging as a Tool to Personalize Respiratory Treatment in Patients With Unilateral Diaphragmatic Paralysis
}

\author{
Cedric S Van Holsbeke PhD, Glenn Leemans MSc, Wim G Vos PhD, Jan W De Backer PhD, \\ Samir C Vinchurkar PhD, Michel Geldof MSc, Pascal R Verdonck PhD, Paul M Parizel PhD, \\ Paul E Van Schil PhD, and Wilfried A De Backer PhD
}

\begin{abstract}
A completely different treatment approach was chosen for 2 patients with unilateral diaphragmatic paralysis and complaints of dyspnea despite similar anatomic and physiologic abnormalities. These decisions were supported by results obtained by functional respiratory imaging (FRI). FRI generated functional information on lobar ventilation and local drug deposition. In the first patient, some lobes were poorly ventilated, and drug deposition simulation showed that some regions were undertreated. This patient underwent diaphragmatic plication to restore ventilation. In the second patient, all lobes were still ventilated. A conservative approach with regular follow-ups was chosen to wait for spontaneous recovery of the diaphragmatic function. Both patients improved subjectively and objectively. These cases demonstrate how novel medical imaging techniques such as FRI can be used to personalize respiratory treatment in patients with unilateral diaphragmatic paralysis. Key words: MeSH; diaphragmatic paralysis; thoracic surgery; pulmonary ventilation; respiratory drug administration; computer-assisted decision making; personalized medicine. [Respir Care 2014;59(9):e127-e131. (C) 2014 Daedalus Enterprises]
\end{abstract}

\section{Introduction}

In the last decade, personalized medicine has received special attention for its potential in individually tailored treatment based on genetic or other information regarding an individual's health status. Following a statement from the European Society of Radiology, medical imaging plays a critical role in all aspects of personalized medicine. ${ }^{1}$

New medical imaging techniques are capable of combining medical images with quantification of certain bio-

Drs Van Holsbeke, Vos, JW De Backer, and Vinchurkar are affiliated with Fluidda nv, Kontich, Belgium. Mr Leemans and Dr WA De Backer are affiliated with the Department of Respiratory Medicine; Dr Van Schil is affiliated with the Department of Thoracic and Vascular Surgery; and Mr Geldof and Dr Parizel are affiliated with the Department of Radiology, Antwerp University Hospital, Antwerpen, Belgium. Mr Leemans and Drs Parizel, Van Schil, and WA De Backer are also affiliated with the Faculty of Medicine and Health Sciences, Antwerp University, Wilrijk (Antwerpen), Belgium. Dr Verdonck is affiliated with bioMMeda, Ghent University, Ghent, Belgium.

Drs JW De Backer and WA De Backer are shareholders and board members of Fluidda nv. Drs Van Holsbeke, Vos, and Vinchurkar are employed by Fluidda nv. The other authors have disclosed no conflicts of interest. logical processes in organs to detect diseases at the earliest possible time, to personalize diagnosis, and to individualize medical or surgical treatment. Functional respiratory imaging (FRI) is a clinically validated computational workflow in which functional data are added to respiratory anatomic images. ${ }^{2}$ Starting with low-dose high-resolution computed tomography (HRCT) scans, 3-dimensional models of airways and lung models are extracted. When performing this extraction on HRCT scans that are taken during breath-holding at 2 (spirometry-controlled) distinct lung levels (functional residual capacity [FRC] and total lung capacity [TLC]), it is now possible to assess the geometric changes in airways and lung lobes during the breathing cycle. These data are then used as boundary conditions for computational fluid dynamics simulations, from which functional information such as flow behavior and in-

\footnotetext{
Mr Leemans presented a version of this paper at the ERS Annual Congress 2013, held September 7-11, 2013, in Barcelona, Spain.

Correspondence: Cedric S Van Holsbeke PhD, Fluidda nv, Groeningenlei 132, 2550 Kontich, Belgium. E-mail: cedric.vanholsbeke@ fluidda.com.
}

DOI: $10.4187 /$ respcare. 02756 
Table 1. Pulmonary Function Tests in Patients 1 and 2

\begin{tabular}{|c|c|c|c|c|}
\hline \multirow{2}{*}{ Parameter } & \multicolumn{2}{|c|}{ Patient 1} & \multicolumn{2}{|c|}{ Patient 2} \\
\hline & Initial Visit & Postoperative & Initial Visit & After $6 \mathrm{mo}$ \\
\hline $\mathrm{FVC}, \%$ predicted & 52 & 61 & 75 & 86 \\
\hline $\mathrm{FEV}_{1}, \%$ predicted & 48 & 57 & 71 & 82 \\
\hline $\mathrm{FEV}_{1} / \mathrm{FVC}$ (Tiffeneau index) & 0.70 & 0.71 & 0.94 & 0.94 \\
\hline $\mathrm{RV}, \%$ predicted & 113 & 96 & 69 & 95 \\
\hline FRC, $\%$ predicted & 77 & 77 & 67 & 94 \\
\hline TLC, $\%$ predicted & 71 & 71 & 71 & 87 \\
\hline $\mathrm{R}_{\mathrm{aw}}, \mathrm{cmH}_{2} \mathrm{O} / \mathrm{s} / \mathrm{L}$ & 6.83 & 4.27 & 3.37 & 2.13 \\
\hline Specific $\mathrm{R}_{\mathrm{aw}}, \mathrm{cmH}_{2} \mathrm{O} / \mathrm{s}$ & 20.69 & 13.36 & 9.88 & 8.26 \\
\hline $\begin{array}{l}\mathrm{FVC}=\text { forced vital capacity } \\
\mathrm{RV}=\text { residual volume } \\
\mathrm{FRC}=\text { functional residual capacity } \\
\mathrm{TLC}=\text { total lung capacity } \\
\mathrm{R}_{\mathrm{aw}}=\text { airway resistance }\end{array}$ & & & & \\
\hline
\end{tabular}

haled medication deposition can be calculated. A detailed description of the FRI methodology was provided by De Backer et al. ${ }^{2}$ One of the design features of FRI is that local analyses can be performed, whereas pulmonary function tests (PFT) are based on a black box approach, in which the information of the whole respiratory system is incorporated into single numbers. This results in increased sensitivity of FRI compared with PFT. ${ }^{3,4}$

In this case report, the clinical value of FRI in personalizing and delivering the most optimal treatment is demonstrated in 2 patients with idiopathic unilateral diaphragmatic paralysis. The FRI workflow is an additional postprocessing step based on paired HRCT scans that are taken as part of routine clinical practice for these types of patients.

\section{Case Reports}

\section{Patient 1}

A 57-y-old male patient $(102 \mathrm{~kg}, 175 \mathrm{~cm})$ with unilateral diaphragmatic paralysis since $1.5 \mathrm{y}$ of age was referred to the hospital with recent complaints of increasing dyspnea and orthopnea. His medical history revealed asthma. On clinical examination, diminished vesicular breath sounds at the right lung base were heard. The previous practitioner prescribed the patient 4 daily doses of salmeterol at $25 \mu \mathrm{g}$ and fluticasone at $250 \mu \mathrm{g}$ (Seretide, GlaxoSmithKline, Philadelphia, Pennsylvania) and fluticasone at $250 \mu \mathrm{g}$ (Flixotide, GlaxoSmithKline) to control the asthma exacerbation. PFT showed highly reduced lung function (Table 1) with both restrictive and obstructive elements. HRCT confirmed unilateral paralysis of the right diaphragm in combination with hypoventilation-induced atelectasis in the right middle and right lower lobes.
To investigate how well the right middle and right lower lobes were still ventilated, FRI analysis was performed. The HRCT scans were loaded into the Mimics 15.0 software suite (Materialise, Leuven, Belgium). This validated package (FDA K073468, Conformité Européenne Certificate BE 05/1191.CE.01) was used to generate 3-dimensional representations of the airways and lobes. The lobar expansion from FRC to TLC was considered a measure for lobar ventilation, as this represents the internal air-flow distribution as defined in the following equation:

$$
\operatorname{IAD}_{\text {lobe }}(\%)=100 \frac{\mathrm{V}_{\text {TLC_lobe }}-\mathrm{V}_{\text {FRC_lobe }}}{\mathrm{V}_{\text {TLC_lungs }}-\mathrm{V}_{\mathrm{FRC} \_ \text {lungs }}}
$$

in which $\mathrm{IAD}_{\text {lobe }}$ is the internal air-flow distribution to a specific lobe, $\mathrm{V}_{\text {TLC_lobe }}$ is the volume of that lobe at TLC, $\mathrm{V}_{\text {FRC_lobe }}$ is the volume of that lobe at FRC, $\mathrm{V}_{\text {TLC_lungs }}$ is the total volume of all lobes at TLC, and $\mathrm{V}_{\text {FRC_lungs }}$ is the total volume of all lobes at FRC. Computational fluid dynamics calculations were performed using Fluent 14.0.0 (Ansys, Lebanon, New Hampshire) and provided measurements of local deposition of inhaled medication. ${ }^{2}$ In this way, it was possible to assess to which zones the drug was delivered.

FRI analysis showed poor ventilation of the right lower lobe and no ventilation of the right middle lobe (Fig. 1). In addition, very low levels of drug deposition were found in these lobes (Fig. 2) when performing particle simulations using the compound data of Tarsin et $\mathrm{al}^{5}$ and Stein et al. ${ }^{6}$ The poor drug deposition results obtained by FRI explain why a combination of several inhaled compounds was needed to treat the asthma, as these zones were probably pharmacologically undertreated. Furthermore, a long-term non-ventilatory status of the right middle lobe could set 


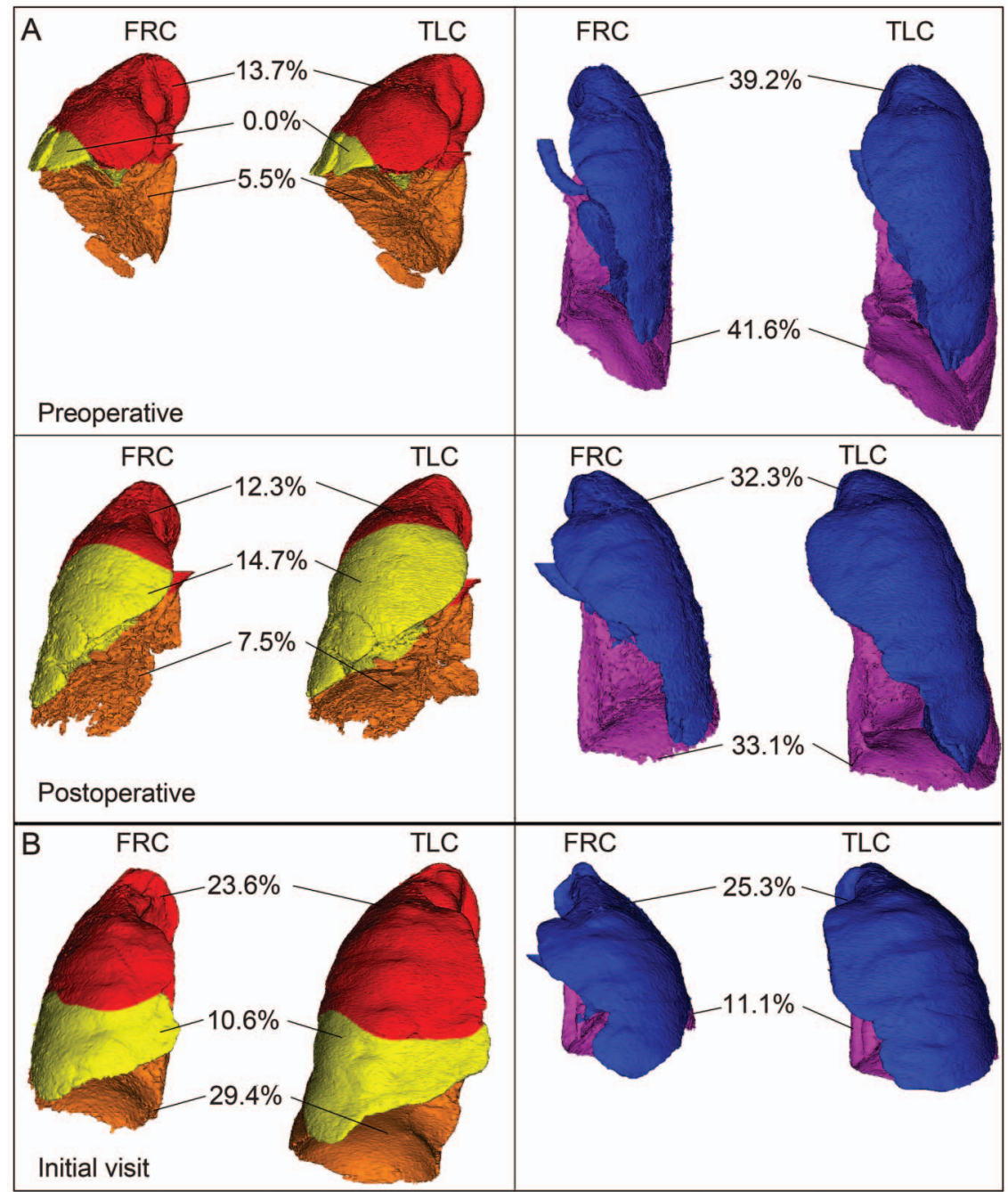

Fig. 1. The internal air-flow distribution in the right upper lobe (red), right middle lobe (yellow), right lower lobe (orange), left upper lobe (blue), and left lower lobe (magenta) seen in patient 1 before and after surgery (A) and in patient 2 (B). FRC = functional residual capacity; TLC $=$ total lung capacity.

the stage for repeated episodes of infection, accounting for a vicious cycle of recurring bouts of inflammation that may result in a non-functional lobe. ${ }^{7}$

In a recent review, Groth and Andrade ${ }^{8}$ concluded that diaphragmatic plication seems to be a promising surgical technique to improve ventilation in patients with diaphragmatic paralysis. Therefore, diaphragmatic plication was performed to place the paralyzed diaphragm in a position of maximal inspiration to relieve compression on the lung parenchyma and to allow its re-expansion.

The dyspnea was subjectively better 6 weeks postoperatively. This was confirmed by an improvement in PFT (see Table 1) and by FRI, which showed that the right middle and right lower lobes were much better ventilated than preoperatively (see Fig. 1). FRI also showed improved drug deposition in these regions (see Fig. 2). The amount of active compound reaching the right middle and right lower lobes increased from $38.2 \mu \mathrm{g}$ (patient using additional fluticasone on top of the salmeterol/fluticasone combination) to $117.1 \mu \mathrm{g}$ (patient only using the salmeterol/fluticasone combination). This was clinically confirmed, as the patient's asthma was kept stable using only 4 daily doses of salmeterol at $25 \mu \mathrm{g}$ and fluticasone at $250 \mu \mathrm{g}$ and as the obstructive components of the PFT improved despite the reduced medication.

\section{Patient 2}

A 57 -y-old male patient $(92 \mathrm{~kg}, 181 \mathrm{~cm})$ with unilateral diaphragmatic paralysis since 11 months of age presented at follow-up with recent complaints of increasing dyspnea and orthopnea. Clinical examination determined diminished vesicular breath sounds at the left lung base. The 


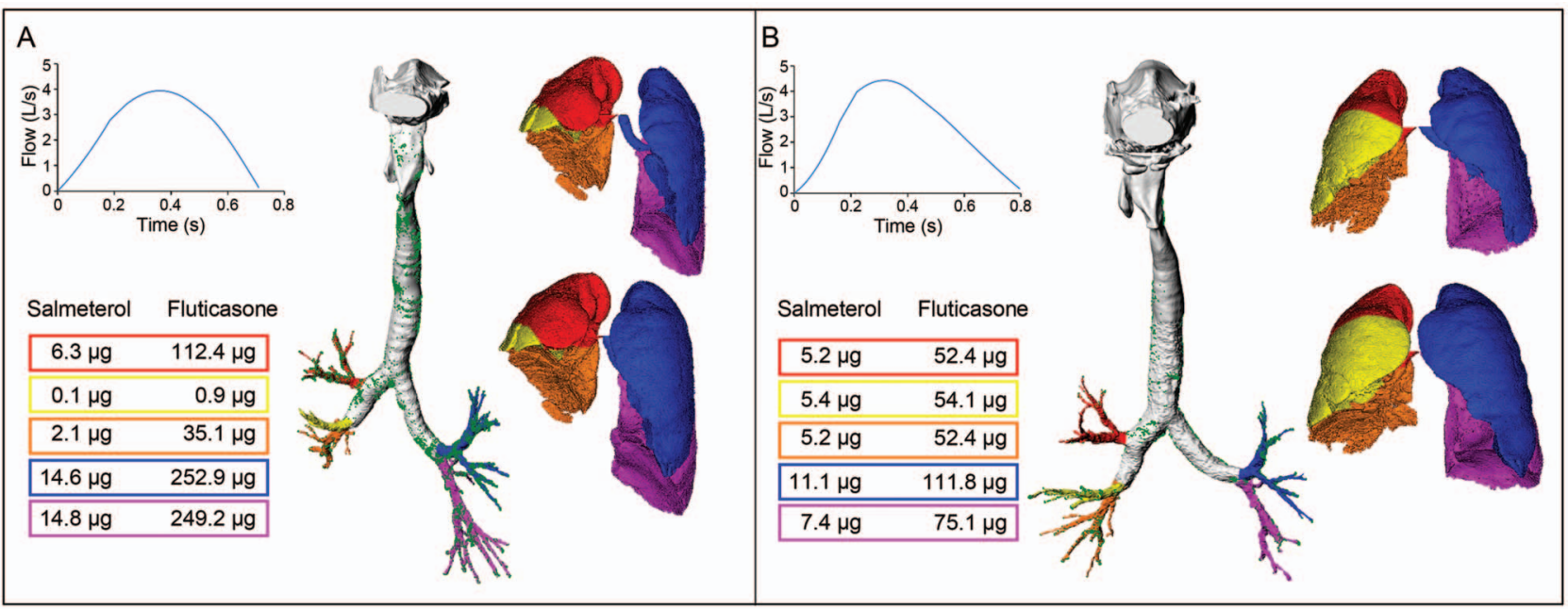

Fig. 2. A visualization of drug deposition simulations of patient 1. The green dots are the locations where the particles deposited. The figure shows how much salmeterol and fluticasone deposited in the right upper lobe (red), right middle lobe (yellow), right lower lobe (orange), left upper lobe (blue), and left lower lobe (magenta). A: Preoperative. B: Postoperative.

patient did not use any inhaled medication. PFT showed significantly reduced lung function (see Table 1) of a restrictive nature. HRCT confirmed the unilateral paralysis of the left diaphragm, and associated atelectasis of the left lower lobe was observed.

Patient 2 underwent the same FRI analysis as patient 1. All lobar regions were ventilated (see Fig. 1). Taking into account these FRI results, the physician considered a further conservative approach with regular follow-up since spontaneous recovery of diaphragmatic function has been reported. ${ }^{9}$ After 6 months, the breathlessness was completely resolved, and TLC was significantly improved (see Table 1). No new FRI analysis was performed, as the physician found this unnecessary from a clinical point of view.

\section{Discussion}

Unilateral diaphragmatic paralysis is characterized by loss of muscle contractility with progressive muscular atrophy that leads to an elevated position of the affected diaphragm. Treatment depends mainly on the cause of the paralysis, anatomic and physiologic impairment (eg, atelectasis), and severity of symptoms. A conservative approach, with or without pharmacotherapy, may be considered since spontaneous recovery of the diaphragm can occur when clinical symptoms are minimal or tolerable and when physiologic impairment is absent. ${ }^{9}$ In those patients with anatomic and physiologic impairment with persisting symptoms despite optimal therapy, surgical correction is indicated. Diaphragmatic plication in a series of 13 subjects with unilateral impairment resulted in an improvement in symptoms, lung function, and quality of life that was maintained over $4-7$ y. ${ }^{10}$ Despite the promising results for both short-term and long-term outcomes, precise selection of suitable patients is necessary. ${ }^{10,11}$

This case report demonstrates the potential of FRI as a new functional imaging technique in respiratory medicine to choose the right treatment plan in patients with idiopathic unilateral diaphragmatic paralysis. In both patients, radiologic examination revealed the appearance of atelectasis in the lower lobes. Atelectasis is commonly seen in combination with compression of the lung parenchyma, which affects compliance, therefore diminishing the regional ventilation of certain parts of the lung. However, traditional clinical techniques are not able to quantify these effects.

Although both patients had the same anatomic and physiologic abnormalities on HRCT, FRI showed a clear difference in regional ventilation. There was no lobar expansion of the right middle lobe and only a minor expansion of the right lower lobe in patient 1 . However, all lobar regions in patient 2 were ventilated. FRI was able to identify functional differences in these 2 patients with apparently the same clinical and radiologic findings.

In addition, FRI was able to calculate the local deposition of inhaled medication. In patient 1, FRI showed that the inhaled medication was not reaching all zones. As a result, the proposed pharmacotherapy was probably inefficient in treating the obstructive symptoms.

Based on the clinical and radiologic results, both patients could be scheduled for surgery because they presented with anatomic and physiologic abnormalities and still experienced symptoms despite optimal treatment. On the one hand, FRI analysis in patient 2 revealed that there was still regional ventilation in the left lower lobe even 


\section{Functional Respiratory Imaging in Patients With Unilateral Diaphragmatic Paralysis}

though atelectasis was present. Therefore, a conservative approach with regular follow-up was chosen, and spontaneous recovery of the diaphragm occurred after 6 months. On the other hand, taking into account the FRI results for patient 1 , we could conclude that surgery was the correct decision for this patient. Finally, this report demonstrates that FRI was able to quantify the regional functional redistribution of air flow to the right lung after diaphragmatic plication, even without major clinical changes in classic lung function tests.

In conclusion, this case report demonstrates the clinical value of FRI as a tool to personalize medical treatment. In the future, this new functional imaging technique may result in more precise diagnosis and treatment of a rapidly growing number of respiratory patients.

\section{REFERENCES}

1. European Society of Radiology. Medical imaging in personalised medicine: a white paper of the research committee of the European Society of Radiology (ESR). Insights Imaging 2011;2(6):621-630.

2. De Backer JW, Vos WG, Vinchurkar SC, Claes R, Drollmann A, Wulfrank D, et al. Validation of computational fluid dynamics in CT-based airway models with SPECT/CT. Radiology 2010;257(3): 854-862.
3. De Backer LA, Vos W, De Backer J, Van Holsbeke C, Vinchurkar $\mathrm{S}$, De Backer W. The acute effect of budesonide/formoterol in COPD: a multi-slice computed tomography and lung function study. Eur Respir J 2012;40(2):298-305.

4. Vos W, De Backer J, Poli G, De Volder A, Ghys L, Van Holsbeke $C$, et al. Novel functional imaging of changes in small airways of patients treated with extrafine beclomethasone/formoterol. Respiration 2013;86(5):393-401.

5. Tarsin WY, Pearson SB, Assi KH, Chrystyn H. Emitted dose estimates from Seretide Diskus and Symbicort Turbuhaler following inhalation by severe asthmatics. Int J Pharm 2006;316(1-2):131-137.

6. Stein SW. Estimating the number of droplets and drug particles emitted from MDIs. AAPS PharmSciTech 2008;9(1):112-115.

7. Albo RJ, Grimes OF. The middle lobe syndrome: a clinical study. Dis Chest 1966;50(5):509-518.

8. Groth SS, Andrade RS. Diaphragm plication for eventration or paralysis: a review of the literature. Ann Thorac Surg 2010;89(6): S2146-S2150.

9. Dernaika TA, Younis WG, Carlile PV. Spontaneous recovery in idiopathic unilateral diaphragmatic paralysis. Respir Care 2008;53(3): 351-354.

10. Celik S, Celik M, Aydemir B, Tunckaya C, Okay T, Dogusoy I. Long-term results of diaphragmatic plication in adults with unilateral diaphragm paralysis. J Cardiothorac Surg 2010;5(1):111.

11. Higgs SM, Hussain A, Jackson M, Donnelly RJ, Berrisford RG. Long-term results of diaphragmatic plication for unilateral diaphragm paralysis. Eur J Cardiothorac Surg 2002;21(2):294-297. 